\title{
Thrombectomy, Lysis, or Heparin Treatment: Concurrent Therapies of Deep Vein Thrombosis: Therapy and Experimental Studies
}

\author{
H. STIEGLER, M.D., H. ARBOGAST, M.D., S. NEES, M.D., \\ A. HALDER, M.D., A. GRAU, M.D., and H. RIESS, M.D.
}

THERAPY

Patients with deep vein thrombosis may be treated in three different ways: by surgical thrombectomy, medical lysis, or simple heparinization. ${ }^{1-3}$ Usually, a certain form of standardization is used for each of the three different therapies. Despite this fact, the question remains: which is the best form of therapy?

In the literature, numerous studies dealing with the problem of the therapy of deep vein thrombosis can be found. A correct evaluation, however, appears extremely difficult because most studies were designed retrospectively, mostly addressing only one form of therapy,,$^{3-5}$ and because results are hardly comparable, since no unique criteria for evaluation of the success of therapy were applied. Many investigators simply relied on the patients' subjective complaints. Others additionally took morphologic criteria into consideration (mostly phlebographic results). Only a few considered functional parameters as important. Therefore, the aim of our study was, to evaluate the validity of each of the three therapies in a global concept. The study was designed prospectively using an ambitious protocol and considering the following three parameters:

1. Clinical history and examination of the patient

2. Morphologic status (such as phlebography)

3. Functional status (such as phlebodynamometry)

From the Departments of Surgery, Physiology, and Medicine, Klinikum Grosshadern, University of Munich, Munich, FRG.

Reprint requests: Dr. Stiegler, Department of Surgery, Klinikum Grosshadern, University of Munich, Munich, FRG.
Patients and Methods

Between January 2, 1984 and July 1, 1986, 233 deep vein thromboses in 209 patients were diagnosed phlebographically at the University of Munich, Klinikum Grosshadern. The form of therapy was determined interdisciplinarily. Thrombectomy was performed under general anesthesia with the preparation of the common femoral vein. For thrombectomy of the pelvic veins, a Fogarty catheter was used obligatorily. Leg veins were thrombectomized by compression. ${ }^{6}$ For lysis, streptokinase (if contraindicated, urokinase) was used at standard dosages, that is, $250,000 \mathrm{IU}$ as bolus within 30 minutes followed by $100,000 \mathrm{IU} /$ hour by perfusor. Heparin therapy was administered by an initial injection of 10,000 IU, followed by continuous infusion using a perfusor and keeping the activated partial thromboplastin time between 80 and 120 seconds. Additionally, all patients were treated with coumarin and compression stockings for 6 months. Sixty-eight (29\%) patients were thrombectomized, 41 $(18 \%)$ were lysed, and $124(53 \%)$ received heparin only (Fig. 1).

Figure 2 shows two phlebodynamograms performed according to the method first described by Kriessmann. ${ }^{7,8}$ The upper tracing is from a healthy patient, the lower one from a patient with post-thrombotic syndrome.

Follow-up investigations were performed 8 days, 3 and 6 months after start of therapy. Results were interpreted according to a clinical score listed in Table 1. In order to reduce the number of invasive procedures, the second follow-up investigation (after 3 months) was performed without a phlebography. 


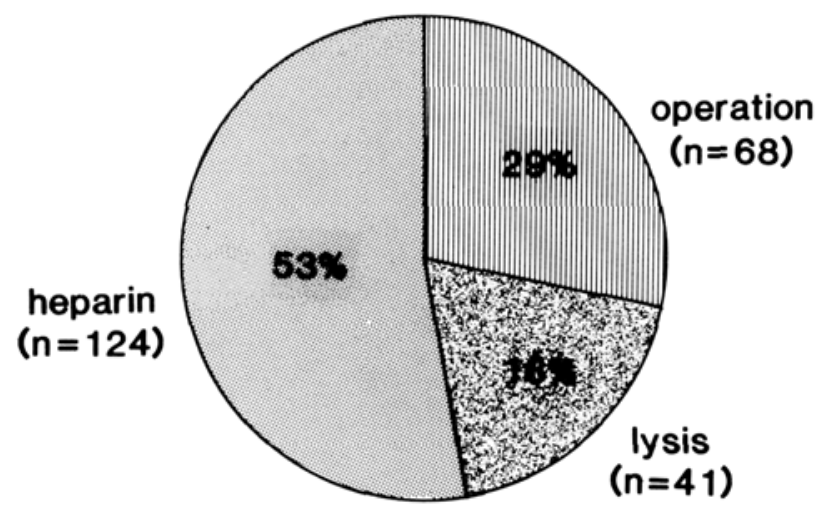

FIG. 1. Distribution of therapies in 233 deep vein thromboses between January 2, 1984 and July 1, 1986.

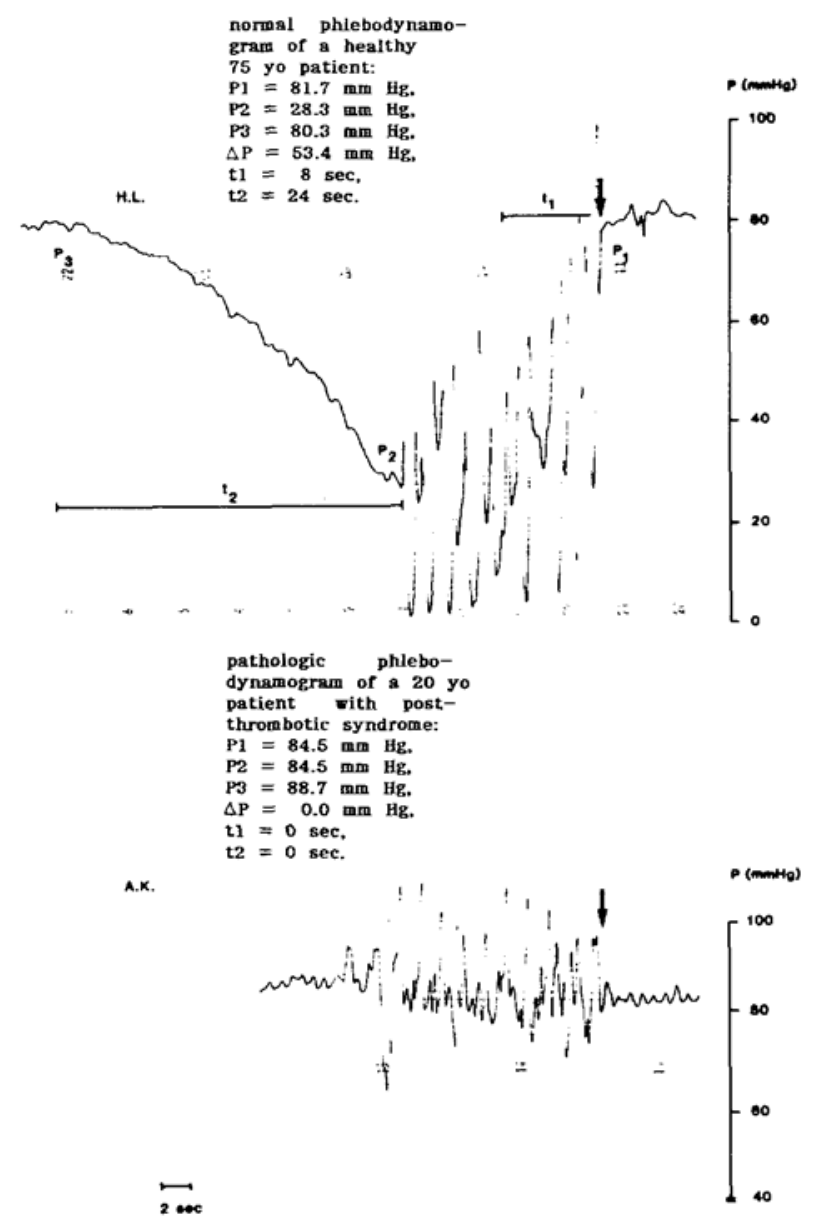

FIG. 2. Phlebodynamometry according to the method first described by Kriessmann in 1974. Puncture of a dorsal foot vein, intravenous pressure measurement by Statham. P1: hydrostatic basal pressure of patient at rest; P2: lowest mean pressure after ten toe standings within 15 seconds; P3: regained basal pressure after ten toe standings; $\Delta \mathrm{P}:$ drop in pressure $=\mathrm{P} 1-\mathrm{P} 2$; $\mathrm{t} 1$ : time of drop in pressure; $\mathrm{t} 2$ : time of pressure rebalance.
TABLE 1. Score for Evaluation of the Success of Therapy of Deep Vein Thrombosis

\begin{tabular}{ll}
\hline \hline Excellent & No complaints \\
& Phlebography: clear \\
& $\Delta \mathrm{P}>50 \mathrm{mmHg}$ \\
Good & No complaints \\
& Phlebography: some residual thrombi \\
& $\Delta \mathrm{P}>50 \mathrm{mmHg}$ \\
Fair & Evening discomfort \\
& Phlebography: impaired drainage \\
& $\Delta \mathrm{P}=35-50 \mathrm{mmHg}$ \\
Poor & All day complaints \\
& Phlebography: impaired drainage \\
& $\Delta \mathrm{P}<35 \mathrm{mmHg}$ \\
\hline
\end{tabular}

\section{Results and Discussion}

The heterogeneity of the patient population can be seen from Figure 3 in which the age distribution for the three therapy groups is demonstrated. It is obvious that more elderly patients underwent heparin therapy than thrombectomy or lysis. This is due to the contraindications for the operative or lytic therapy, age itself being one (only exception is age greater than 65 years). Only 5.8 versus $4.8 \%$ of all patients in the thrombectomy or lysis group, respectively, had malignant disease, whereas the corresponding number for the heparin treatment group was $29.8 \%$. Also the location of the thrombosis underscores the heterogeneity of the patient population (Fig. 4). Patients with pelvic vein thrombosis (mostly combined with thigh and lower leg thrombosis) were most commonly subjected to surgery. Patients with isolated lower leg thrombosis were most often treated with heparin. Lysis was favored in distal forms of venous thrombosis. The contraindications for operative and lytic therapy are listed in Table 2. The partly differing frequencies indicate the different judgments of the participants of the interdisciplinary study. In many patients, more than one contraindication was present (Table 3). Again, the large variety of contraindications and their different distribution underscores the divergence of the therapeutic groups. Of the large number of patients with contraindications for thrombectomy or lysis only 39 for patients with venous thromboses $(16.7 \%)$ could have been treated with either thrombectomy or lysis (Fig. 5). Therefore a concurrence between these two therapies can hardly be stated.

The value of the different forms of therapy of deep vein thrombosis can partly be elucidated from Figure 6 , in which the results of the follow-up investigations 6 months after start of treatment are compiled. It is apparent that surgery and lysis yielded better results than heparin therapy alone. Still, in the heparin group, $22 \%$ demonstrated excellent late results. Further analysis revealed that the patient population had exclusively partial lower leg 
TABLE 2. Contraindications ( $\mathrm{Cl}$ ) for Surgery and Lysis and Their Encountered Frequency in 233 Vein Thromboses

\begin{tabular}{lrlr}
\hline \multicolumn{1}{c}{ Surgery } & $\%$ & \multicolumn{1}{c}{ Lysis } \\
\hline Location of thrombosis & 22 & Previous operation & 26 \\
Age of thrombus & 21 & Malignant disease & 18 \\
Malignant disease & 17 & Age of the patient & 16 \\
Age of the patient & 16 & Hypertension & 13 \\
Contraindications for anesthesia & 7 & Age of thrombus & 13 \\
Pulmonary embolism & 5 & Location of thrombosis & 11 \\
& & Intramuscular puncture sites of trauma & 10 \\
& & Central nervous system trauma or operation & 7 \\
& & Gastric or duodenal ulcers & 5 \\
& & Diabetes mellitus & 4 \\
& & Hemorrhages & 3 \\
& & Pregnancy & 4 \\
\hline
\end{tabular}

vein thromboses. Apparently, the fibrinolysis mediated by the endothelium was sufficient for lysis of the relatively small masses of thrombi.

The data shown in Figure 6 represent the largest patient population so far reinvestigated by the invasive and ambitious protocol. Nevertheless, the low absolute number of patients reinvestigated after 6 months limits the statements that can be made from our data. The main reason for the limited number of reinvestigations was a persistent immobilization, which made phlebodynamometry impossible. Rarely, patients refused phlebography. In the heparin group, $20 \%$ lethality was

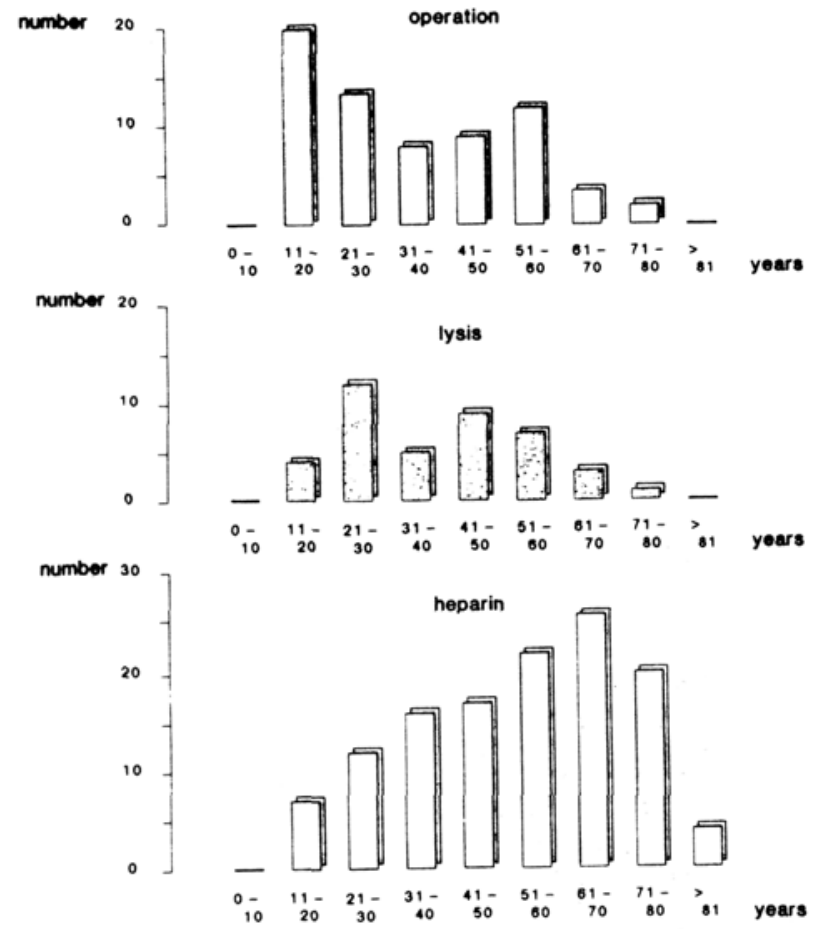

FIG. 3. Age distribution of all patients with venous thromboses subdivided by form of therapy. observed, almost exclusively as a result of malignant disease. When the results of the 39 venous thromboses without contraindications for surgery and lysis are compared, both therapies show almost equally good results (Fig. 7). When excellent and good results are combined, the numbers are 53 and $58 \%$ for thrombectomy and lysis, respectively. Again, the exact interpretation of the results is restrained by the different locations of the thromboses in the different treatment groups. (In the surgical patients the combined pelvic, thigh, and lower leg vein thromboses were most frequently encountered). No good results with heparin alone were achieved in patients with combined

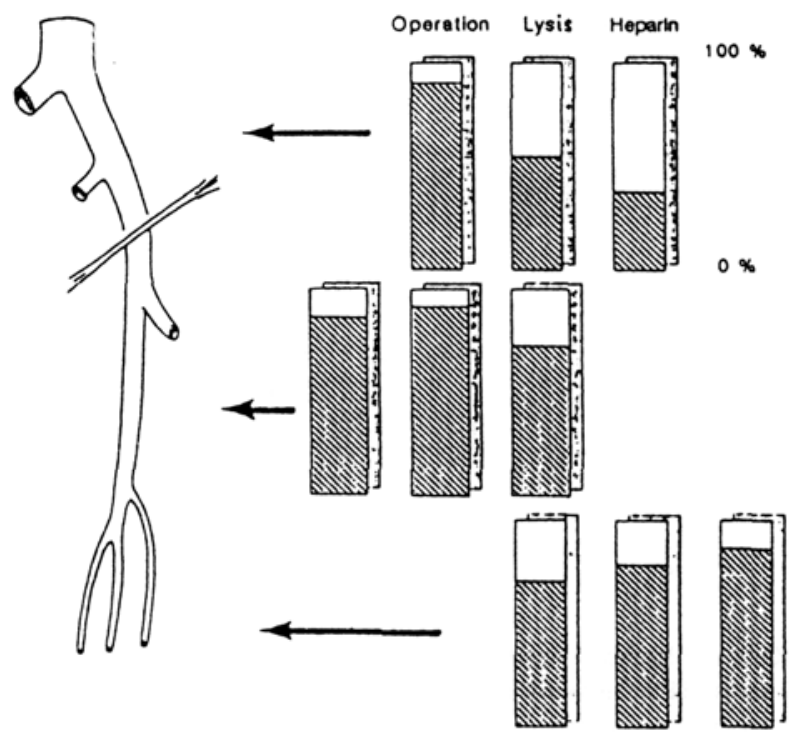

FIG. 4. Frequency of location of thrombosis at the pelvic, thigh, and lower leg level in percent of the total number of respective treatment group. 
TABLE 3. Number of Contraindications for Surgery and Lysis

\begin{tabular}{ccc}
\hline $\begin{array}{c}\text { No. } \\
\text { Contraindications }\end{array}$ & $\begin{array}{c}\text { Surgery } \\
(\%)\end{array}$ & $\begin{array}{c}\text { Lysis } \\
(\%)\end{array}$ \\
\hline 1 & 41 & 32 \\
2 & 18 & 24 \\
3 & 3 & 13 \\
4 & 0 & 2 \\
5 & 0 & 1 \\
\hline
\end{tabular}

pelvic, thigh, and lower leg vein thromboses when the process was 8 days or older before the onset of therapy. In comparison to the latter group of patients, with 55\% excellent or good outcome, operative therapy gave the best results. With lysis comparable results were obtained in only $20 \%$ of the patients. Again, the low number of cases must be taken into consideration (Fig. 8).

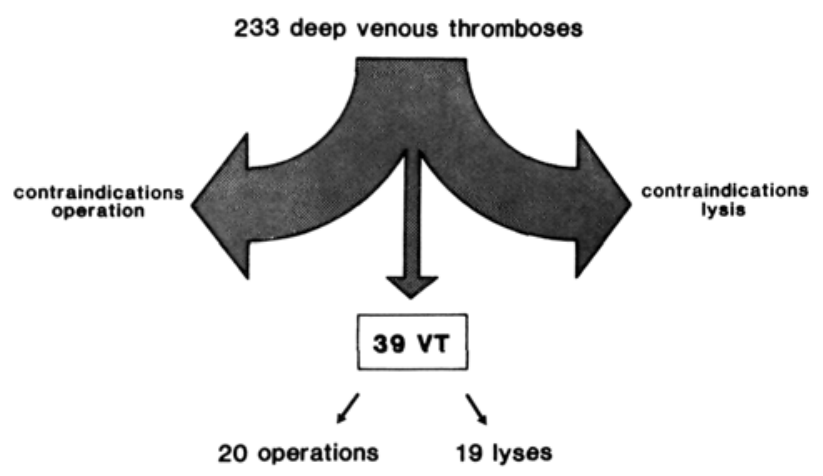

FIG. 5. Only 39 of the 233 incidences of deep vein thromboses could be treated either surgically or by lysis.

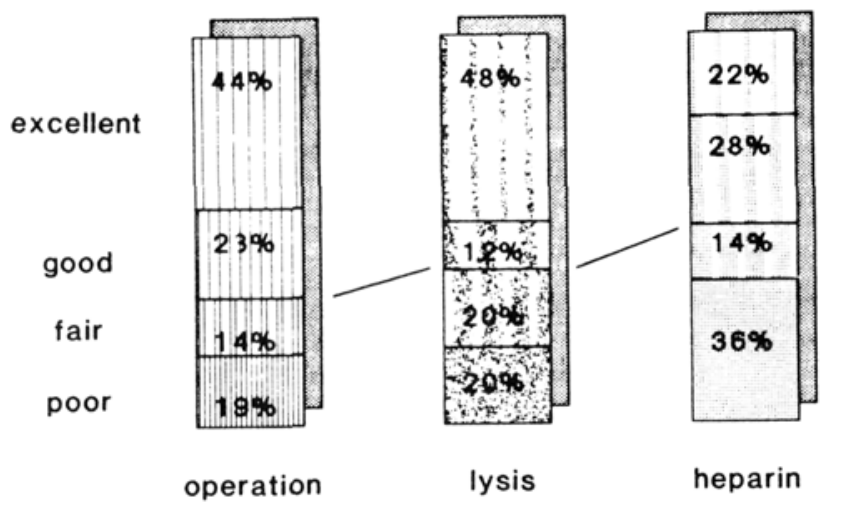

FIG. 6. Results 6 months after therapy of deep vein thrombosis. Evaluation corresponding to the scores depicted in Table 1 (33 operations, 20 lysis, 41 heparin).

\section{Conclusions}

A study comparing the results of different therapies of deep vein thrombosis has always to consider the heterogeneity of the patient population. The establishment of better comparable subgroups always results in a diminution of the total number of cases, hence limiting the reliability of the outcome. This is also applicable to this investigation. Nevertheless, the present data may be used as aids in the process of clinical decision-making. Heparin therapy is only indicated in an isolated thrombosis of the lower leg. In cases of combined thromboses in different compartments, lysis and surgery clearly reveal better results, but contraindications to thrombectomy and lysis must always be considered. In these patients therefore heparin treatment alone cannot be looked on as an adequate therapy. In combined pelvic, thigh, and lower leg thromboses, thrombectomy seems to be the most

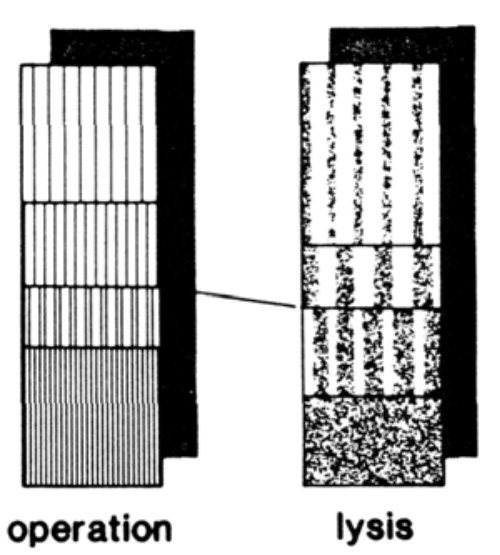

FIG. 7. Results 6 months after therapy of deep vein thrombosis. No contraindications for surgery and lysis ( 16 operation, 10 lysis).
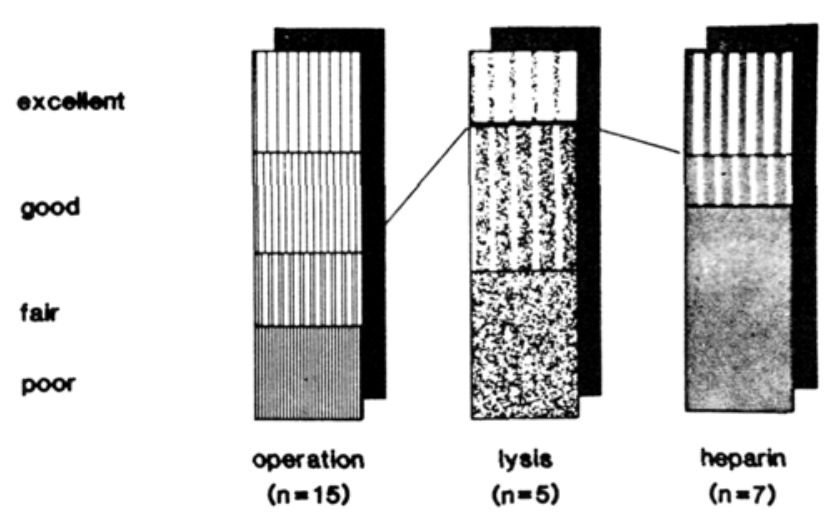

FIG. 8. Results 6 months after onset of therapy in patients with combined pelvic, thigh, and lower leg thrombosis. Thrombus age less than 8 days. 
promising form of treatment. Despite these guidelines for therapy of deep vein thrombosis, even with the aggressive forms of treatment (surgery and lysis), no completely satisfactory results can be obtained, still leaving a $40 \%$ incidence of postthrombotic syndrome. Thus, the question remains unanswered whether an additional approach might improve the present therapeutic possibilities. Our main interest has focused on platelets, which have so far been widely neglected from consideration in the venous system. This fact is difficult to understand, since thrombosis per se provokes endothelial damage, hence exposing an impaired vessel wall to the blood after successful treatment of thrombosis. Therefore, we studied in an animal experiment whether inhibition of platelet aggregation, already known to be effective for the arterial endothelium, also works on the venous vessel wall and, if so, whether this mechanism can be verified by endothelial damage.

\section{EXPERIMENTAL STUDY OF THE ANTITHROMBOGENICITY OF THE VENOUS WALL}

Among numerous complex metabolic functions, the normal vascular endothelium also demonstrates antithrombotic properties by inhibiting coagulation, ${ }^{9,10}$ activating fibrinolysis, ${ }^{11}$ and inhibiting platelet aggregation (Fig. 9 a). From investigations with arterial endothelial cell cultures, it is known that endothelial cells continuously secrete prostacyclin $\left(\mathrm{PGI}_{2}\right)$ into the plasma, which inhibits platelet aggregation. This process is modified by numerous metabolic pathways, such as thrombin significantly increasing the release of $\mathrm{PGI}_{2} \cdot{ }^{12,13}$ The endothelial cells can provide additional inhibition of platelet aggregation through a continuous secretion of adenosine into the plasma. Adenosine inhibits platelet aggregation independently from $\mathrm{PGI}_{2}$. In addition, the endothelial cells have a complete ectonucleotidase cascade, which rapidly degrades the accruing adenine nucleotides, adenosine triphosphate (ATP), adenosine diphosphate (ADP); both stimulating platelet aggregation, thereby accumulating the metabolite adenosine intraluminally. ${ }^{14,15}$ Such a release of ATP or ADP is the result of any cell moult and vessel wall trauma. Locally, concentrations as high as $10^{-2} \mathrm{M}$ can be reached (Fig. 9 b). The aim of our investigation was to evaluate whether these metabolic pathways are of any importance in the venous endothelium and, if so, to what extent are they altered by thrombosis. Since these questions could not solely be addressed by investigations of endothelial cell cultures, an individual animal experiment was designed, allowing the study not only in an isolated perfused vessel, but also providing thrombosis experiments in vivo.
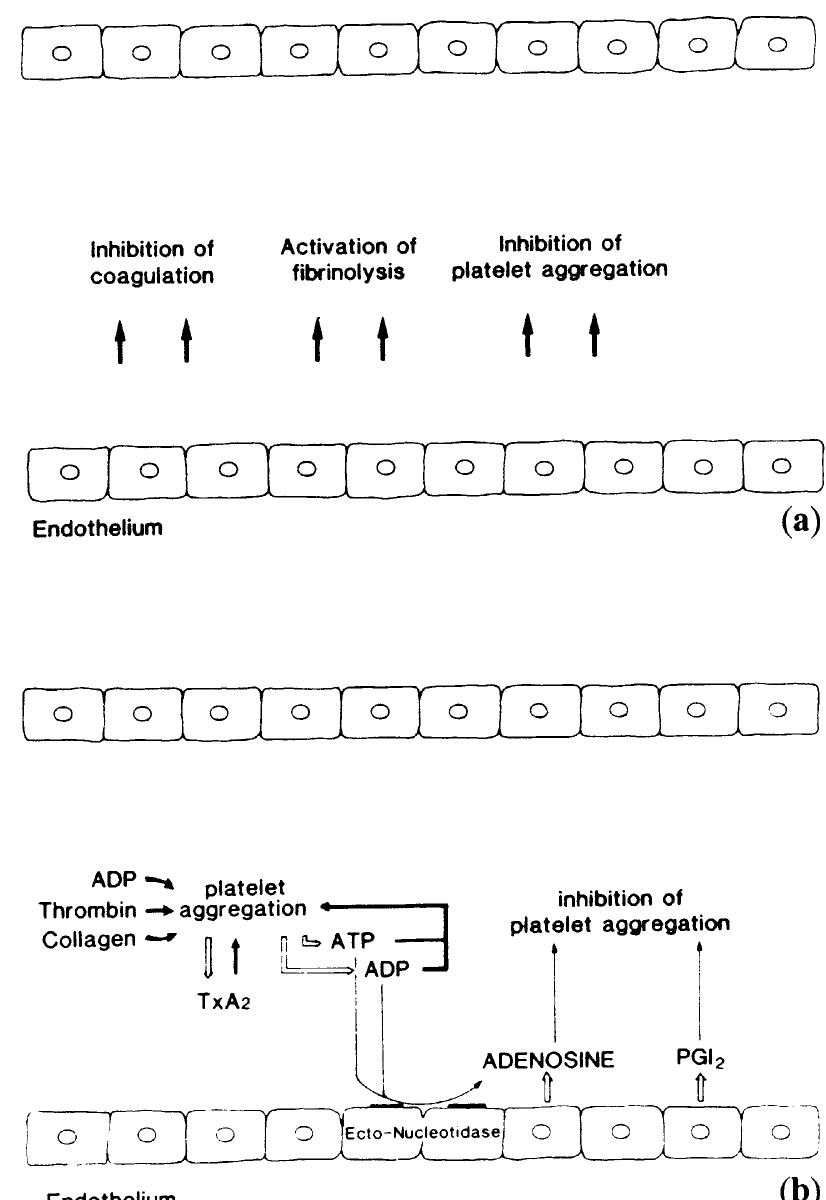

FIG. 9. a: Schematic representation of the endothelial properties preventing thrombosis. $b$ : Schematic representation of physiologic, endothelium-mediated inhibition of platelet ${ }^{18}$ aggregation. (Modified according to Gerlach et al).

\section{Materials and Methods}

Isolated vena cava segments of rabbits were used for our experiments. The animals were anesthetized by ketamine and xylazine. In order to simulate the endothelial damage caused by thrombosis, before explantation, thrombin was applied by a catheter transfemorally to the infrarenally clamped vena cava. The resulting thrombus was conserved by an additional distal clamp. Some of the thrombosed segments were thrombectomized after 1 hour; for chronic thrombosis, the thrombus was fixed by ligations for 8 days and thrombectomized by a Fogarty catheter. After explantation, isolated vena cava segments were perfused under standardized conditions, with modified Earle's salt solution and HEPES buffer (Fig. 10). For investigations of reendothelialization, explantation was performed in some animals 14 days after thrombectomy of an 8-day-old thrombus. 


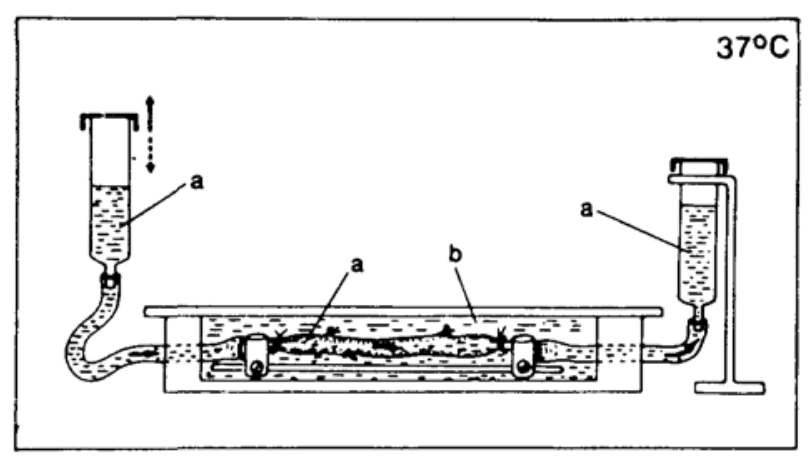

FIG. 10. Isolated perfused vena cava segment. a: perfusion medium; b: external medium.

After completion of the perfusion studies, vena cava segments were prepared for scanning electron microscopy. Similarly, the structure of some thrombi was investigated. ${ }^{16}$ The activities of ectonucleotidases of the venous wall were determined by the metabolization of nucleotides (only shown for ADP) found in the perfusate $\left(10^{-4} \mathrm{M}\right)$. Measurements were performed by high- performance liquid chromatography analysis. ${ }^{17}$

\section{Results}

Figures 11 to 13 show pictures of endothelium in different shapes, obtained by scanning electron microscopy (SEM). Figure 11 depicts normal venous endothelium after 8 hours of standardized perfusion, indicating no damage by the perfusion procedure itself. Figure 12 clearly illustrates the luminal wall changes of chronically thrombosed veins ligated for 8 days. Drastic morphologic changes, such as completely deendothelialized venous wall segments, indicate the effect of longer lasting thrombosis on the vessel wall. Figure 13 shows a venous wall initially heavily damaged by a thrombus ( 8 days old) after 14 additional days of regeneration. The initial morphologic and physiologic changes (denudation of the vessel wall) have improved, the signs of proliferating cells are already visible, clearly indicating the regenerative capabilities of a previously damaged venous wall. Figure 14 summarizes the results of our measurements of metabolites of nucleotides in isolated perfused vena cava segments with normal endothelium (a), after thrombosis for 1 hour and thrombectomy by irrigation without instrumental manipulation (b), and after thrombosis for 8 days and thrombectomy by Fogarty catheter (c). As the figure indicates, compared with control experiments, thrombosed (for 1 hour) venous endothelium expectedly dephosphorylizes ADP rapidly. Adenosine production, however, is clearly decreased. This tendency to diminished adenosine accumulation during metabolism

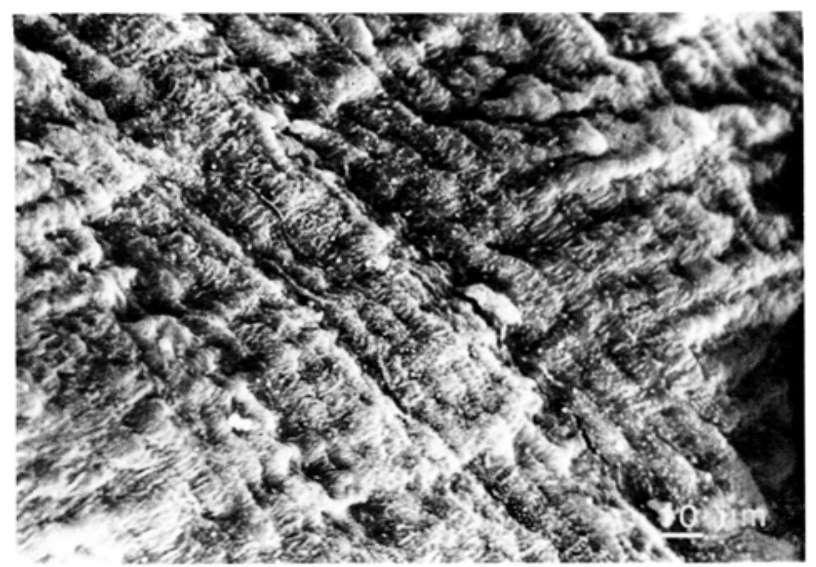

FIG. 11. Scanning electron microscopic picture of venous endothelium, after 8 hours of standardized perfusion. Intact endothelium is covering all of the subendothelial matrix.

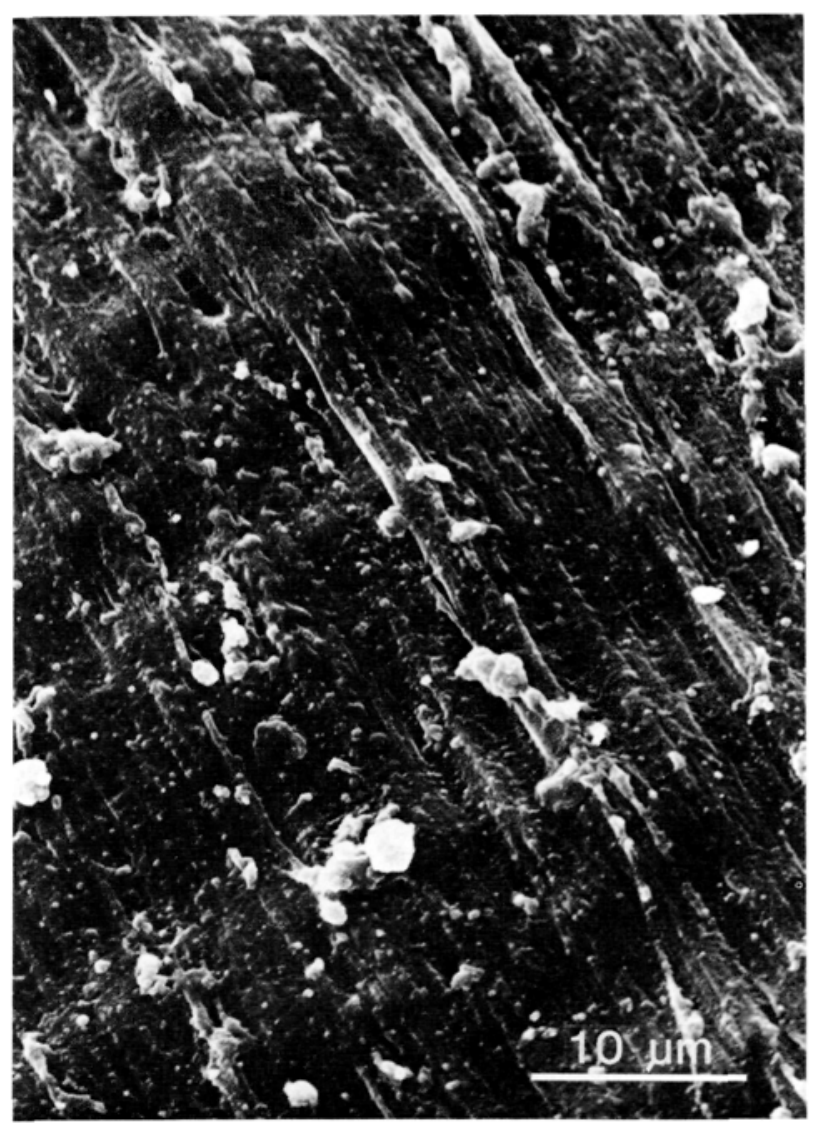

FIG. 12. Scanning electron microscopic picture of a vena cava segment after 8 days of thrombosis and thrombectomy. 


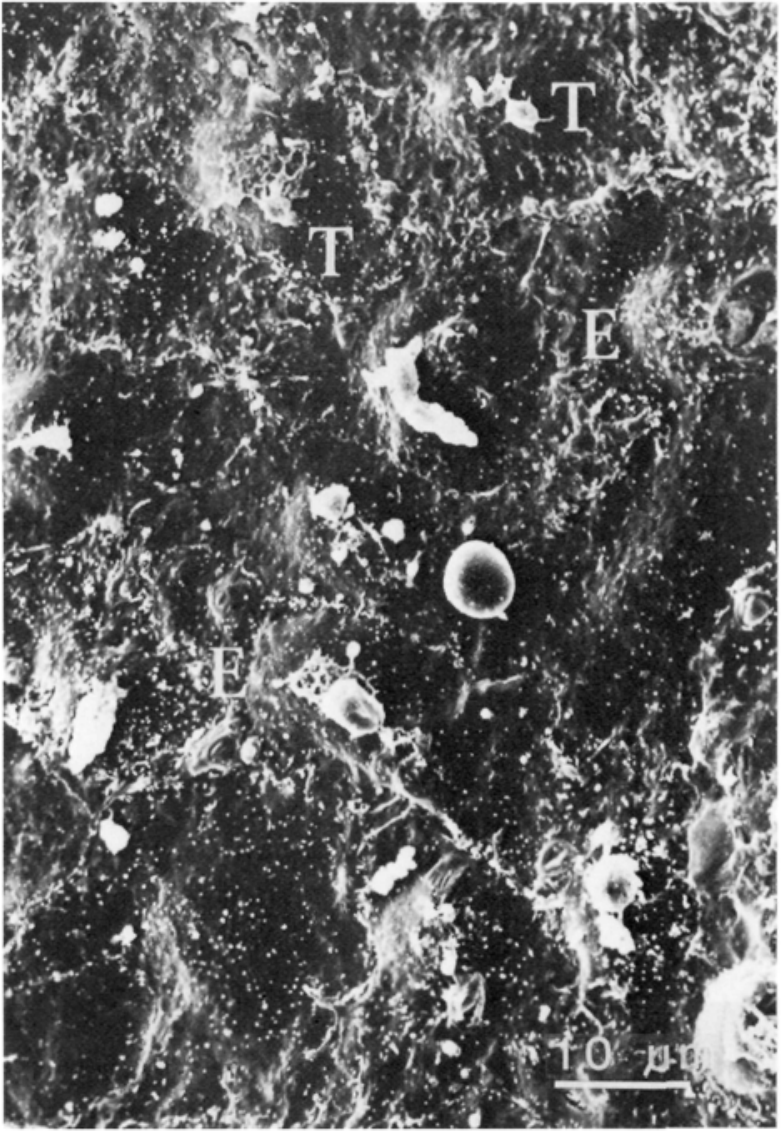

FIG. 13. Scanning electron microscopic presentation of the intima of a vena cava segment after thrombosis for 8 days, thrombectomy, and 14 days of restitution. Among cell detritus, between proliferating endothelial cells $(E)$, numerous walladherent thrombocytes $(\mathrm{T})$ are discernible.

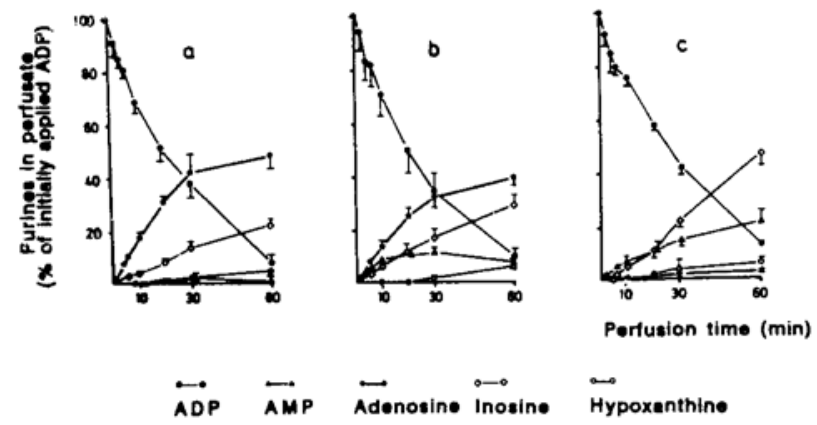

FIG. 14. Degradation of $\operatorname{ADP}\left(10^{-4} \mathrm{M}\right)$ in isolated perfused vena cava inferior segments of four rabbits ( $\pm \mathrm{SE}$ ). a: with intact endothelium (control); b: with thrombosis in situ (1 hour) and thrombectomy; $\mathrm{c}$ : with thrombosis in situ ( 8 days) and thrombectomy. of intraluminally applied ADP is drastically improved in the experiments with a thrombosis age of 8 days. Under such circumstances, adenosine hardly accumulates, revealing inosine as the typical main degradation product.

The changes in the intraluminal degradation of ADP could be due to different reasons and are not easily explained. In order to evaluate the extent of damage caused by the Fogarty catheter used for thrombectomy, the thrombus was also extracted by simple irrigation with PBS solution, excluding a direct instrumental trauma to the vessel well. The degradation of ADP revealed almost exactly the same results as depicted in Figure 14. This seems to suggest that the damage already caused by thrombosis alone is so extensive, that thrombectomy hardly causes a significant additional injury.

The results of the nucleotide determination in reendothelialized vena cava segments are shown in Figure 15. ADP is rapidly dephosphorylized. Interestingly, adenosine monophosphate accumulated in the medium. Unlike the thrombosed and solely thrombectomized (without regeneration) veins, similar to the control vessels of untreated animals after reendothelialization of the venous segments, adenosine again represented the main fraction of dephosphorylized metabolites, again functionally supporting the morphologic findings.

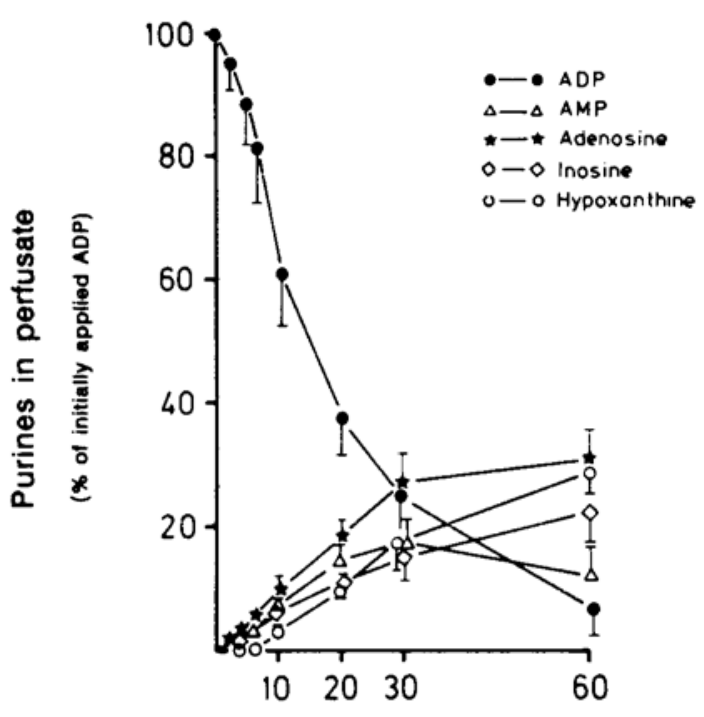

Perfusion time (min)

FIG. 15. Degradation of $\operatorname{ADP}\left(10^{-4} \mathrm{M}\right)$ in isolated perfused vena cava segments of rabbits, after thrombosis in situ ( 8 days), thrombectomy, and reendothelialization in situ (14 days). 


\section{Discussion}

Venous endothelium is provided with a complete ectonucleotidase cascade (Fig. 16 a), which rapidly degrades accrued adenine nucleotides to adenosine, inhibiting platelet aggregation. The degradation products inosine and hypoxanthine are inactive concerning platelet aggregation and emerge after cellular uptake of adenosine. If the integrity of the venous wall is damaged, liberation of adenosine desaminase takes place, rapidly degrading adenosine to inosine (result of special experiments, not shown). There is hardly any intermediate adenosine formed (Fig. 16 b). Hence, after venous endothelial damage, degradation of ATP or ADP by ectonucleotidases

\begin{tabular}{lll|l|l|l|l|l|l|l|l|}
\hline 0 & 0 & 0 & 0 & 0 & 0 & 0 & 0 & 0 & 0 \\
\hline
\end{tabular}

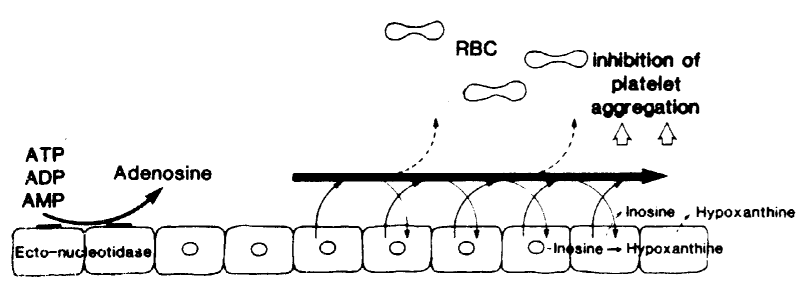

Endothelium

(a)

\begin{tabular}{|c|c|c|c|c|c|c|c|c|c|}
\hline 0 & 0 & 0 & 0 & 0 & 0 & 0 & 0 & 0 & 0 \\
\hline
\end{tabular}

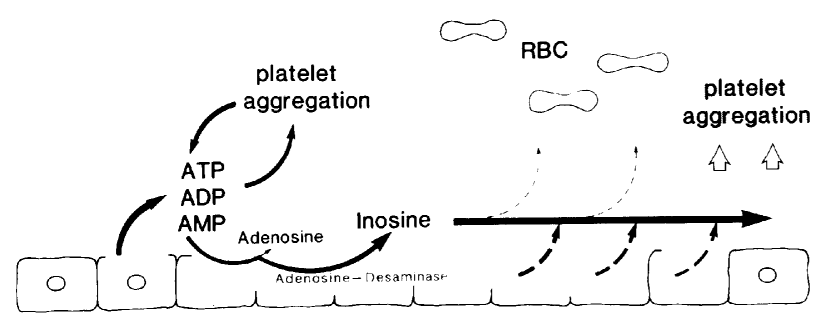

Endothelium

(b)

FIG. 16. a: Venous endothelium and its antiaggregatory properties: complete ectonucleotidase cascade. (Modified according to Gerlach et al. ${ }^{18}$ ) b: Consequences of endothelial damage: platelet aggregation by degradation of adenosine. remains unchanged, compared with the intact vessel. By the lack of adenosine accumulation, the second possibility of effectively inhibiting platelet aggregation has vanished, thus causing a shift toward platelet aggregation.

\section{Conclusions}

Even by applying the most sophisticated modern possibilities of surgical procedures, a striking improvement in the therapy of deep vein thrombosis can only be expected when our understanding of the pathogenesis improves, thus enabling the physician to look for more promising forms of therapy. In this context, it seems important to recognize, that not only surgical thrombectomy with a Fogarty catheter, but also thrombosis itself causes damage to the vessel wall favoring rethrombosis. In the experimental study, the venous endothelium could be proved to have a significant quantitative effect on the degradation of nucleotides favoring platelet aggregation. Chronic thrombosis already causes fundamental endothelial damage. During reendothelialization, a normalization of nucleotide metabolism can be observed. For the clinical practice, the following consequences can be summarized:

1. Prophylaxis of thrombosis has to be performed aggressively, preventing the origin of a deep vein thrombosis.

2. In case of deep vein thrombosis, lysis offers an endothelium-saving procedure in fresh venous thromboses; in case of contraindications, thrombectomy may still be the method of choice.

3. In case of older venous thrombosis, treated by lysis or operation, the reduction of antiaggregatory mechanisms has to be considered. Future therapeutic efforts have to be directed toward additional antiaggregatory substances.

\section{REFERENCES}

1. Huk J, T Hölzenbein, W Pichler et al: Langzeitergebnisse nach chirurgischer therapie der bein-beckenvenenthrombose. Vasa 20 (Suppl):145-149, 1987.

2. Persson AU, KM Ekdahl: Treatment of acute deep venous thrombosis with fibrinolytic agents. Med Clin North Am 70:1325-1332, 1986.

3. Trübestein G: Can thrombolytics prevent post-phlebitic syndrome and thrombo-embolic disease? Hemostasis 16 (Suppl 3): 38-50, 1986.

4. Holm HA, B Finnanger, A Hartmann et al: Heparin treatment of deep venous thrombosis in 280 patients: Symptoms related to dosage. Acta Med Scand 215:47-53, 1984.

5. Stiegler H, U Tosch, L Sunder-Plassmann: Venöse thrombektomie iliofemoraler venenthrombosen. Munch Med Wochenschr 127:84-87, 1985. 
6. Stiegler H, L Sunder-Plassmann: Akute Verschlüsse der Venen. In:Heberer G, RJAM van Dongen, Eds: Kirschnersche Allgemeine und Spezielle Operationslehre, Gefäßchirurgie. Springer, Berlin, 1987.

7. May R: Messmethoden in der Venenchirurgie. Verlag Hans Huber, Bern, 1971.

8. Kriessmann A: Periphere Phlebodynamometrie. Grundlagen, Technik, Leistungsbreite. Vasa 4 (Suppl):1-35, 1975.

9. Barnes MJ, DM Scott: Glycoproteins secreted by the endothelium and their involvement in specific interactions at the subendothelium. In:Cryer A, Ed: Biochemical Interactions at the Endothelium. Elsevier, New York, 1973, p. 111.

10. Oohira A, TN Wight, P Bornstein: Sulfated proteoglycans synthesized by vascular endothelial cells in culture. J Biol Chem 258:2014-2021, 1982.

11. Loskutoff DJ, E Levin: Properties of plasminogen activators produced by endothelial cells. In: Jaffe EC, Ed: Biology of Endothelial Cells. Martinus Nijlhoff, Boston, 1984, pp. 200-208.

12. Higgs, EA, S Moncada, JR Vane: Prostacyclin $\left(\mathrm{PGI}_{2}\right)$ inhibits the formation of platelet thrombi induced by adenosine diphosphate (ADP) in vivo. Br J Pharmacol 61:137, 1977.
13. Weksler BB, CW Levy, EA Jaffe: Stimulation of endothelial cell prostacyclin production by thrombin, trypsin and the ionophore $\mathrm{A}$ 23187. J Clin Invest 62:923-930, 1978.

14. Caen JP, CS Jenkins, H Michel, J Pokutecky, R Bellanger: Adenosine degradation products, platelet adenosine uptake and inhibition of aggregation. In:Caprino L, E Rossi, Eds: Platelet Aggregation and Drugs, Academic Press, New York, 1974, pp. 129-141.

15. Pearson JD, JS Carleton, JL Gordon: Metabolism of adenine nucleotides by ectoenzymes of vascular endothelial and smooth muscle cells in culture. Biochem J 170:265-271, 1978.

16. Hayat MA: Fixation for Electron Microscopy. Academic Press, New York, 1981.

17. Stiegler H: Antithrombogenität der Venenwand: Klinische Erfahrungen und Experimentelle Studien. Habilitationsschrift zur erlangung des grades eines habilitierten Doktors der Medizin an der Ludwig-Maximilians-Universität, München, 1987.

18. Gerlach E, BF Becker, S Nees: Formation of adenosine by vascular endothelium: A homeostatic and antithrombogenic mechanism? In:Gerlach E, BF Becker, Eds: Topics and Perspectives in Adenosine Research, Springer, Berlin, 1987, pp. 209-222. 